\title{
Reflection and practice on generative music structuring: the formal problem of ergodicity
}

\author{
Gabriel Montechiari e Silva, Clayton Rosa Mamedes \\ Department of Arts - Federal University of Paraná \\ Rua Coronel Dulcídio, 638 - 80420-170 Curitiba, PR \\ g.monte.silva@gmail.com, claytonmamedes@gmail.com
}

\begin{abstract}
This article approaches the subject of musical form from the standpoint of an algorithmic composition practice. It introduces the problem of ergodicity in music, a formal situation at which music development is perceived as static.

The concepts of General Periodicity by Henri Pousseur and Temporal Gestalt by James Tenney gave support to a reflection on the nature of the problem, as well as to formulate a twofold structuring procedure based on ideas of continuity and segmentation of the musical course. The devised method was implemented as a computer program to produce entire pieces of music.
\end{abstract}

\section{Introduction}

The research presented in this paper is part of a project to develop an automated system of composition that encompasses the production of entire musical pieces. All computer programs we created thus far are parts of a single poietic vision, rather than tools to assist the compositional process. They are the compositional process. That being said, on each step of this enterprise a particular issue was brought forth - always based on a subjective evaluation from the composer's point of view. Here we will discuss a formal situation that could be characterized as 'structural silence', and refers to the moment at which a musical piece sounds repetitive.

Curtis Roads pointed out that "one of the great unsolved problems in algorithmic composition is the generation of coherent multilayered structures: mesoform and macroform" [5]. However, the structural problem is not discussed at length in the literature on algorithmic composition. The bibliography on computer music often exhibits a descriptive and catalographic posture. Books like Computer Music [2], and the more specific Composing Music With Computers [3], and Algorithmic Composition $^{I}$ [4], provide detailed information about the techniques applied on computer generated music, but they rarely reflect on the musical issues that arise from their implementation. Gerard Nierhaus acknowledged this issue when he observed that "composers publishing

\footnotetext{
${ }^{1}$ To make justice, Nierhaus does reflect on algorithmic composition on the conclusion of that book, and also does a remarkable job in his later book Patterns of Intuition.
}

material on their algorithmic methods is rather a rare occurrence". He presents some hypothesis to understand why that is, suggesting, for one, that it might be because of a "fear of 'disenchantment' at a piece's creative merit" a magician never reveals his tricks. Also, algorithmic composition "is mostly only used as a supplementary tool in genuine composition" - this means that by not employing automation throughout the composition process, issues like this do not occur.

Another point made by Nierhaus is that "procedures of algorithmic composition are [...] mainly used in the field of style imitation" [4], meaning that the focus of these works is in assessing how well the techniques perform in replicating an aesthetic. Then, any flaw in macroform or a broader musical context is pointed out as an issue of the computational method, rather than compositional. In other words, the worries of researchers in the latter kind of works is not that of a composer, but of a musicologist, and their reports reflect that.

The few papers that provide subjective analysis often do so by the use of ambiguous remarks. In this respect, we observed various terms employed to reference the structural problem in algorithmic composition. For instance G. Papadopoulos and G. Wiggins, referring to stochastic methods, stated that "it is difficult to capture higher or more abstract levels of music" [6]. In a similar fashion, Nierhaus pointed out that "in most cases, [a stochastic model] is not able to describe the context sufficiently"[4].

In the same sense, while analyzing the results generated by the program WolframTones, Curtis Roads stated that those "start without formal opening and continue mechanically ad infinitum, without taking into account higher forces operating above the sonic surface", and evaluated that "these banal sound patterns [...] operate on only one level of organization; they lack narrative macrostructure, development, sectional organization, and macroform" [5]. The term 'banal' was used almost 60 year prior by R. C. Pinkerton to refer to the lack of structural content in melodies generated by the use of transition tables [1]. In all these cases, the authors refer to the inability of the models in dealing with different hierarchical levels of musical form.

The deficiency we've been talking about is also referred to as a lack of musical meaning. Papadopoulos 
and Wiggins pointed out that some of the techniques used in algorithmic composition "do not make any strong claims about the semantics of the pieces". According to them generative grammars, as an example, "generate a large number of musical strings of questionable quality". The authors stated that "the big disadvantage of most, if not all, the computational models (in varying degrees) is that the music that they produce is meaningless" [5].

Roads suggested that "musical meaning is embedded in layers and encoded in many simultaneous musical parameters or dimensions" [6]. In that sense, we could make a claim in favor of the interpretation that musical meaning emerges from perceiving and responding to the internal relations of a musical piece [7]. And, if "the meaning communicated by a formal system is, essentially, itself [...], a 'tying-together' of ideas and experiences" [8], talking about the lack of meaning would be equivalent to talking about the lack of formal structure.

As pointed out earlier, this structural problem is related to a sense of repetitiveness, but the repetition we refer to is of a special kind. This is not a literal repetition, but an abstract one, that arises from the mechanics behind the ordering of a piece. Silvio Ferraz [9] approached the problem of repetition through referencing some of twentieth century's musical streams. In serial music, specifically, he identified a kind of repetition resulting from "the unifying principle of the material diversity that these works presented". The author said that "if we understand repetition as a notion that extends to whichever element that returns in a given system, [...], we could say that the repetition in serial thought is given as a conceptual repetition" [9]. With the idea of conceptual repetition in mind, we can glimpse at why the formal stasis problem is constantly lurking behind an algorithmic composition work.

Algorithms are lists of procedures, rules. It has been said that "a rule or law signifies a finite or infinite procedure, always the same, applied to continuous or 'discrete' elements", and "this definition implies the notion of repetition, of recurrence in time" [10]. So, "in order for a rule to exist it must be applicable several times in eternity's space and time", and a periodic property is implied. It would seem, then, that there is no escaping repetitiveness if we are to use algorithmic means of composition.

While there is little written about that in algorithmic composition literature, the way to deal with the two poles of boredom (by excess of variety, or by lack thereof) was widely discussed by twentieth century composers - specially by those on the Darmstadt school. We studied theoretical works of two post-war composers, Henri Pousseur and James Tenney, and through their interpretation on the nature of musical structure we devised and implemented an automated composition method focused on the meso and macro structural level of musical forming.

\section{The General Periodicity and Temporal Gestalten}

Henri Pousseur, while analyzing Structures by Pierre Boulez, questioned why that highly organized serial piece sounded just like one composed by aleatoric processes. To him, this work presents "movements deprived [...] of any individual meaning" [11] (we may remember the terms employed on the criticism of algorithmic processes pointed out at the introduction).

According to Pousseur, a characteristic of total serial music is that, "exception made to the measures and rules themselves, nothing seem to be let to the care of free intent", however, if we focus only on perception without analytical means - "what we experience is precisely the opposite of this kind of ordering. Precisely in cases in which the most abstract constructs are applied, often we have the impression of being before the workings of some aleatoric principle". In that sense, a piece offers "great resistance to unitary apprehension and distinctive memorization", characteristic to which Pousseur associates the term 'equiprobability', referring to a situation in which "at any moment, anything can happen, at least to our perception" [11].

In Structures, the distribution of the material constantly exploits all the variation span. Boulez consciously reached for extreme unpredictability, and he stated "his intention to manifest time's irreversibility, and that means [...] a negation of periodicity, no matter how varied it may be" [11]. This strategy served an ideal of abolishing all musical hierarchy - in this case taken to the last consequence. We may say that in the total serial experiment "the elimination of hierarchies in the realm of simple sound parameters made controlling the contrasts difficult", what resulted "in leveling and consequent complete stasis of an even sound surface" [9].

In the music of Anton Webern, Pousseur identified a case of particular equilibrium between the ethos of non-polarization and formal clarity. To him, the Austrian composer have succeeded where the next generation have not, and the reason was that Webern "refused to oppose to the course of time", and "definitely recognized its autonomy" [11]. According to Pousseur, the overwhelming material differentiation has a suppressing effect on our perception of time, what is directly linked to stasis, since "time is synonym of movement". The author connected this to a wave-like character of the structural/formal issue, by stating that "movement can only be [...] alternated. We always evolve in a finite space; when a transformation develops in a particular direction, it finds sooner or later a concrete boundary, to it unsurpassable" [11]. Then, it would only be possible to ensure a constant impression of movement by means of some level of undulatory development of the musical course. Therefore, music that is fruit of a conscious effort to banish all kind of periodicity would be doomed to formal stagnation.

Behind the idea of general periodicity is the notion that every aspect of musical structuring can be interpreted as a wave that modulates a base material. The 
superposition of complex parametric waves, at different hierarchical levels, establishes a connection between the material and the abstract variation field in which it is set. Pousseur's theory considers the undulatory phenomenon under the perspective of an electronic music composer. In that sense, the author speaks about different waveforms, amplitude and frequency modulation, and other concepts that comes from his studio practice. Electronic music prompted the idea of applying compositional procedures to sound generation - the composing of sounds -, but also allowed for the opposite idea. According to Pousseur's theory, musical composition could be generalized as being like audio synthesis. From this perspective, he considered the problem that we've been talking about.

A melodic arch, with a well defined directionality, gives us the impression of half a structural cycle, with a clear wavelength; while the disposition of pitches (or some other parameter) in a constellation-like manner hinders the estimate of an unambiguous wavelength. As Pousseur suggests, our ability of distinguishing parametric periods is linked to the perception of musical structure. Thus, in the latter case, the listener would be unable to sense how the material was structured. It could be said that structural silence is similar to white noise and, as our conscience tends to suppress a noise after being exposed to it for some time, we would also alienate ourselves before a formal situation like that. This metaphor contemplates the other extreme of periodicity, with perception suppressing a prolonged single tone.

The solution proposed by Pousseur for keeping a clear formal development lies on the parsimonious use of the variation range in each parameter. According to him, "if we want to produce a wave that is effective in all respects, a wave that establishes unity, it will be necessary [...] an economic use of the elementary units that define the variation space"[11]. The composer advocates the conscientious use of periodicity, not its complete suppression, because periodicity and aperiodicity are complementary.

We notice that, in terms of stasis, Pousseur refers especially to a chaotic formal situation, and this is not the case for many melodies obtained by generative techniques. However, the reason for stasis in an algorithmic music context remains the same as in Pousseur's case: in continuous generation, there will be a point at which all the variation boundaries were already explored - even if, combinatorially, the process keeps producing different material. In that sense, excessive reiteration of an algorithm equates to a drone.

While to Henri Pousseur musical structures behave like waves, to James Tenney they behave like particles. Tenney's article Temporal Gestalt Perception in Music starts with a comparison of two distinct conceptions of time: "for the historian, time is not the undifferentiated 'continuum' of the theoretical physicist, but a hierarchically ordered network of moments" [12]. To Pousseur, musical time was something continuous (as he related structure to continuous functions). To Tenney there is a hierarchical structure that sections time in contextual regions, he stated that "for the musician, a piece of music does not consist merely of an inarticulate stream of elementary sounds, but a hierarchically ordered network of sounds, motives, phrases, passages, sections, movements, etc.". In other words, musical time consists of "time-spans whose perception boundaries are largely determined by the nature of the sounds and sound-configurations occurring within them". These time-spans have the characteristic of being "both internally cohesive and externally segregated from comparable time-spans immediately preceding and following it". Tenney called these time units 'temporal gestalt-units' (TGs).

His article comes from a quest to objectively delineate the borders of the structural groups we form during music perception. Since the book Meta+Hodos [13] Tenney has been speculating about adapting the principles of Gestalt psychology (as it was applied to visual stimuli) to the domain of sound. Thus, borrowing the gestalt concepts of similarity and proximity, the author established a structural unit called clang, defined by this statement: "in a collection of sound-elements, those which are simultaneous or contiguous will tend to form clangs, while relatively greater separations in time will produce segregation, other factors being equal. Those which are similar (with respect to values in some parameter) will tend to form clangs, while relative dissimilarity will produce segregation, other factors being equal" [12]. However, on his 1980 article, Tenney looked for creating a formal method of analysis that could be automated and generalized to other structural levels.

The first step Tenney took towards the quantification of his gestalt analysis was a change in the methodological approach. There was a "shift of emphasis from the unifying effects of proximity and similarity to the segregative effects of temporal separation and parametric dissimilarity" [12]. Allied to that, was the strengthening of the notion that any process based on perception should be considered in terms of the passing of time, and not as an 'out of time' analysis. In relation to this last concept, he concluded that the delimitation of clangs should be based solely on the identification of the starting point of a new one, as listening proceeds. This point is determined based on a local maxima on the parametric gap between elements. In other words, the point at which a parametric interval is bigger than the interval prior and after it, that's where a new clang should start.

To get to the final version of his model, Tenney needed a way of unifying all the parametric intervals in just one measure of distance. To do that, the author established a metric space. He considered this one of the most important steps to the development of his model: "to treat musical space as a metric space within which all the individual parametric intervals between two points might be integrated into a single measure of distance" [12].

A metric space is a mathematical concept that refers to an ordered pair $(M, d)$, constituted of a set of spatial coordinates $M$ and a metric $d$ (a function to 
calculate the distance between two points from the set associated with it).

The last step before the generalization of his model could extend it to hierarchical levels beyond the clang was deciding on a method to pinpoint the position of gestalt units of a higher level. In summation, Tenney used the centers of mass of each collection of elements.
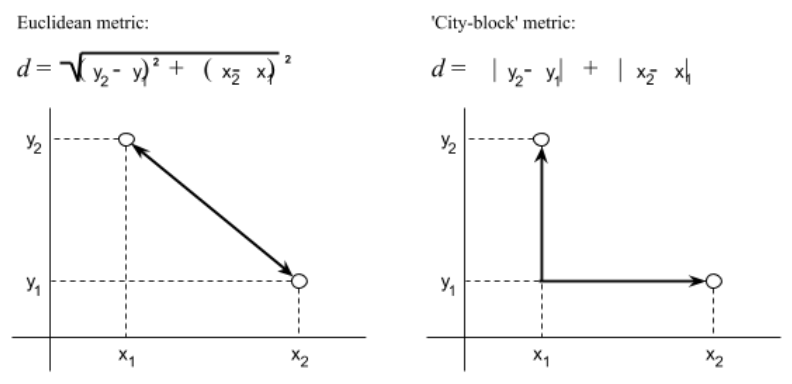

\section{Figure 1: Two examples of metric (adapted from Tenney, 1980, p.213)}

The last formulation of his model was: "a new TG at the next higher level will be initiated in perception whenever a TG occurs whose disjunction (with respect to the previous TG at the same hierarchical level) is greater than those immediately preceding and following it".

Based on his theory of temporal gestalten, Tenney noted the same problem tackled by Pousseur: "serial, aleatoric and stochastic compositional methods frequently result in textures which are statistically homogeneous at some fairly low hierarchical level. A typical negative response to this kind of formal situation (which I have elsewhere called 'ergodic') is that, although 'everything is changing, everything remains the same.' [12]. His paper proposed a way of dealing with this situation.

During our research, we often used the term 'ergodicity' to designate the structural problem we've been facing. The word Tenney chose originates from its homonymous applied to the study of dynamic systems. Independently, Iannis Xenakis briefly addressed a definition to the term, that helps us illustrate how it could be applied on a musical context: "the ergodic principle states that the capricious effect of an operation that depends on chance is regularized more and more as the operation is repeated" [10]. In other words, it deals with situations in which a reiterated random process tends to some regularity. In the same way, a reiterated compositional procedure would be subject to this phenomenon. Another reason to highlight the term 'ergodicity' is to explicitly reference Tenney's work, by which he designates a measurable situation. To Tenney, "a piece becomes 'ergodic' (with respect to some parameter) as soon as a hierarchical level is reached [...] at which the mean-intervals between successive TGs are all effectively zero" [12]. If there are not discrepancies in the distance between structures, a new level is not reached and the piece embarks on a static state - an ergodic formal situation.

As we've seen, Henri Pousseur believed the structural problem is caused by prolonged and equanimous exploration of the variation space. James Tenney reached a similar diagnosis, and pointed out that "the more the total available range in some parameter is 'used up' at a given level, the smaller will the average effective difference between TGs at that level, and more quickly will the texture approach 'ergodicity' at the next higher level". Be it through a nexus of complex waves, or through a system of mass points, both authors arrived at the same conclusion in this matter.

\section{Our practice}

We associated Pousseur and Tenney with two aspects of musical structure: continuity and segmentation. Pousseur's theoretical work have continuity as a theme, since - even if manifested discreetly - structure was pictured as an abstract wave, a continuous phenomenon. On the other hand, Tenney's work was about distances as structural determinants, segmentation was his theme. We envisioned a twofold structuring procedure combining ideas from both authors.

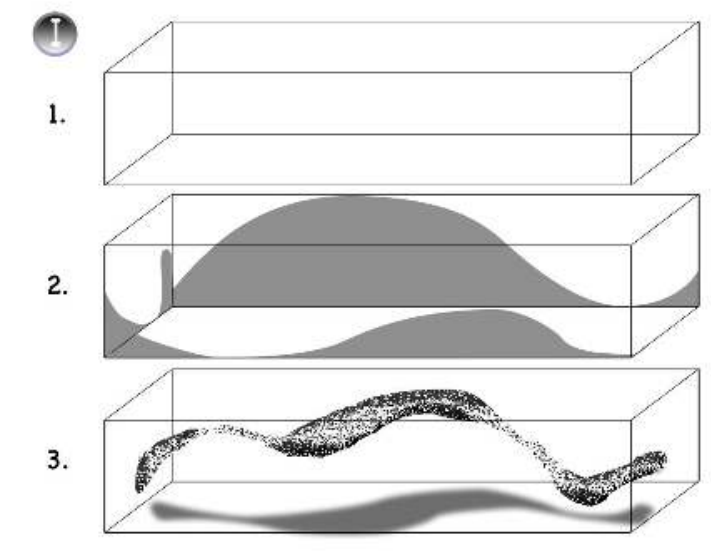

Figure 2: The first step in our compositional model to institute a continuity in musical space.

The first step in our compositional procedure was focused on establishing continuity. We started by defining a space in which our form would develop (Fig. 2-1). Values of absolute duration, pitch register, and dynamic range, define the fringes of this space. At this stage, two issues remain: which metric to use, and which weights - if any. The metric will determine the way by which we calculate distances, and the weights are the factors by which we redimension the axes. On our implementation, we used euclidean metric and empirically estimated the weights. As these are speculative points on Tenney's theory, we made sure that different options could be easily tested. There is also a good reason weighting remained a variable: Tenney noticed that each piece analyzed by his method required a different set of weights to optimally correspond to the manually performed analysis. In that sense, the author 
suggested that the discrepancies in weight values between analysis of different composers were due to differences in the structural role which parameter had on given composer's aesthetic.

Next, parametric profiles are defined (Fig. 2-2), whose function is to modulate the material in a continuous curve in space (Fig. 2-3). This means establishing a conducting line to the musical course. There were profiles to four parameters: global dynamics, pitch transposition, tempo, and a fourth parameter referring to melodic variation. These profiles' shapes are sinusoids that, accordingly to the principle of even exploration of the variation space, have wavelengths corresponding approximately to the duration of the piece - slightly varying on each instantiation. The initial phases of each profile vary in a uniformly random manner.

This simple procedure seemed to prevent the feeling of stasis. However, demarcations were lacking between regions of space. Each area of the musical space represents a distinct musical character due to the parameters assigned to each axis, so the continuous transition between them hinders the apprehension of the specifics of form - despite its existence being clearer due to the parsimonious exploration of space. A second procedure was made for sectioning the form, thus emphasizing distinct shapes and the order in which they appear.

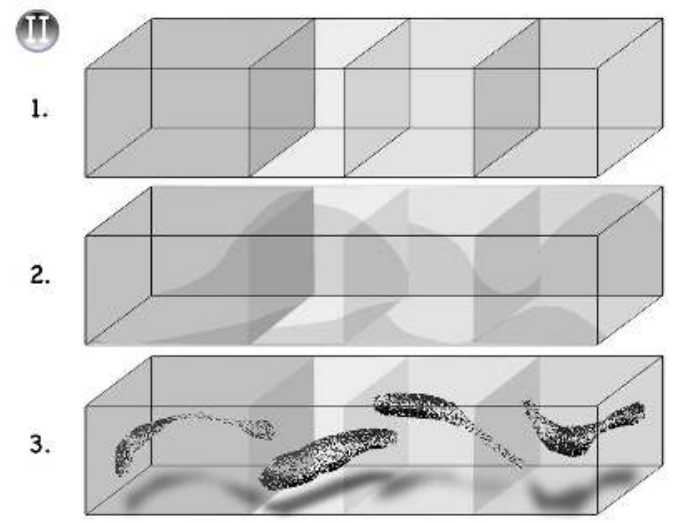

\section{Figure 3: Second step in our compositional model - to break the continuity.}

The procedure to introduce structural marks was conceived according to the following principle: they could not derive from material factors - i.e., from repetition of previous material or from introduction of fundamentally distinct material. Our solution was based on the physical phenomenon of refraction. When an object passes through two transparent media, we can observe the boundary between them through the discontinuity of the object's image. Inspired by this, we determined the creation of sections with different refractive indices (Fig. 3-1), and depending on the slope of the parametric profile at the point of junction between sections, the profile's phase is shifted accordingly (Fig. $3-2)$. The discontinuity thus produced introduces points of reference that help us on the characterization and ordering of distinct moments in a piece (Fig. 3-3).

There is yet a third step, whose function was to promote adjustments on the resulting structure. Its first part consisted of optimizing the distance between structures (Fig. 4-1). For that, it was applied the optimization technique of differential evolution, with a fitness function that judged sets of candidate section displacements, choosing the set with the furthest apart sections. By considering every distance between structures (and not only those between contiguous ones), we avoided a situation in which there is only contrasting structures but no global variation - i.e., we ensured they do not just alternate in space. As a consequence, the exploration of unique zones in space means promoting musical passages with unique characters, what led us to a more effective formal distinction - here, there was a double operation of unifying (by continuity) and differentiating (by segregation) musical structures.

The melodic material we used came from a generator made on previous works, and have a particular microstructure. The aim of the second adjustment was to emphasize this microstructure (Fig. 4-2). It starts with a structural analysis utilizing Tenney's method, followed by intensity accentuation at the points where a TG starts. This brings up the low-level hierarchy of a section.

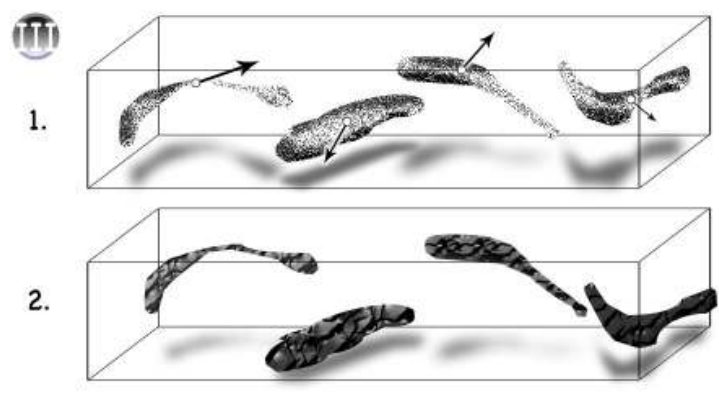

Figure 4: Third step in our compositional method fine tuning of structure distances, globally [1], and locally [2].

Below we have a picture of five piano-rolls of pieces generated by our program (Fig. 5). Based on them we can make a brief morphological analysis of the process' results. The pitches are on the vertical axis, time is on the horizontal axis, and the intensities are represented by the opacity of each note. Each piece has a particular duration, but some of the images were squashed to fit a uniform column.

The first item (Fig. 5-a) has the clearest section distinctions. There is little register directionality on each section. However, the sections are well spread on this axis. The contrast on the average dynamics contribute to the easy distinction. Tempo ${ }^{2}$ is the only attribute that don't seem to contrast between sections, but is a strong

\footnotetext{
${ }^{2}$ Our pieces don't have metrics, 'tempo' is just a multiplier to the durations of the base material.
} 
conveyor of directionality on this example.

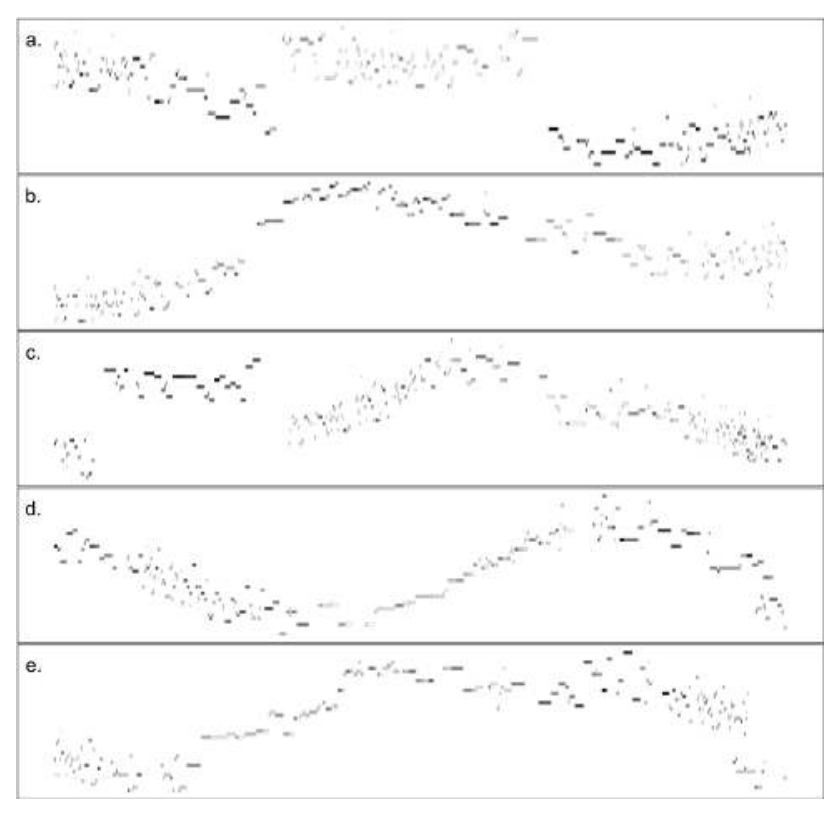

Figure 5: Visual representation of five pieces produced by our program.

An important thing to notice is that some instances don't show a clear sectioning. This happens because, as we mentioned, our method of segregation was inspired by refraction and depending on the angle a parametric profile 'hits' a new section, its phase will be shifted more or less. In item $d$ (Fig. 5-d), the sinusoid shape of the profiles is most apparent, and at first glance it seems like there is no sectioning. The reason for this is that the section boundaries are located close to the trough and the crest of the pitch profile, and that causes a smaller shift in that parameter. However, each parameter has a different profile, so they have different displacements between sections, and we can still deduce structural boundaries based on some of them. This is the case of item $b$ (Fig. 5-b), where there are also three sections. Between the first two, there is a gap in register, but between the middle and the last section there appears to be a single downward motion on the overall register, and the structural limits would be blurred. However, if we look closely there is a contrast on the average dynamics of both sections, so we can still spot those limits.

\section{Conclusion}

Our long term objective is the conception of an automated compositional system for producing entire pieces of music. However, until now we were only able to produce musical fragments lacking in high level structure. Given the complexity of the musical phenomenon, there's been moments at which we thought that a complete musical piece couldn't be achieved through automated methods. Now, for the first time since the beginning of our practice, we reached a result that exhibits some structural 'completeness'. Although aesthetically there is much to be done, we concluded that the minimum requirement for complete pieces was met.

At a composer's perspective, the biggest insight our research brought was the realisation that increasingly complicated methods were not the way to overcome the complexity barrier. The formal/structural problem don't require intricate intellectual constructs. It can be greatly simplified by considering it a matter of distributing objects in musical space. Some spatial configurations convey greater complexity than what is needed to describe them. On the other hand, often when these configurations are assembled in a strict logical manner, the complexity is lost in translation to apparent randomness - as it was pointed out by Pousseur, referring to examples in total serialization.

At the end of this research we were left with two guiding principles for structuring music; and one prescription to avoid structural silence, a static formal situation that Tenney called ergodic. The principles relate to the concepts of continuity and separation, two sides of the same coin. The prescription is to populate the variation space in an economical fashion.

In the field of algorithmic composition there is an attraction towards ideas such as fractal geometry, evolutionary algorithms, cellular automata, neural networks, etc. At times we are tempted to believe that the solutions to our compositional problems will come from technical novelties. Computers make possible to apply meticulous calculations to musical creation, thus allowing us to go further as composers. However, being able to go further means nothing if one doesn't know where to go. Our algorithms are not subject to asymptotic analysis; we don't owe tribute to operational efficiency. Our practice won't benefit from sophisticated computing techniques if there is no solid representational basis supporting it.

The conceptual framework we rely on, and the idea we make of what music is, these are the real origins of the methods we work with as composer-programmers. Computer scientists will focus on algorithmic innovation. To us is left the elaboration of compositional systems capable of manifesting pieces of music in compliance to our inner necessity - even when this necessity is not previously formalized, as in cases where the model and its implementation are built simultaneously. May this not be mistook as a claim for pure intuition, because our métier absolutely depends on the formalized method. The point we make is that the method of interest to us a particular one: it is the creative method.

"May our imagination rouse our intelligence, and may our intelligence ensure our imagination." [14]

\section{Acknowledgements}

This research was supported by the CAPES Higher Education Improvement Coordination.

\section{References}

[1] HILLER, L. A.; ISAACSON, L. M. Experimental 
Music. Nova York: McGraw-Hill, 1959.

[2] DODGE, C.; JERSE, T. A. Computer Music: synthesis, composition and performance. $2^{\mathrm{a}}$ ed. Estados Unidos: Schirmer, 1997.

[3] MIRANDA, Eduardo Reck. Composing Music with Computers. Burlington: Focal Press, 2002.

[4] NIERHAUS, Gerard. Algorithmic Composition. Alemanha: Springer-Verlag, 2009.

[5] PAPAdopoulos, G.; WIGGINS, G. AI Methods for Algorithmic Composition: A Survey, a Critical View and a Future Prospects. School of Artificial Intelligence, Division of Informatics. Edimburgo: Universidade de Edimburgo, 2000.

[6] ROADS, Curtis. Composing Electronic Music. Nova Iorque: Oxford Press, 2015.

[7] MEYER, L. B. Emotion and Meaning in Music. Chicago: Chicago Univ. Press, 1956.

[8] COELHO DE SOUZA, R.; FARIA, R. A Criatividade como Propriedade Emergente na Composição Algoritmica. SONIC IDEAS. 10. 21-34, 2013.

[9] FERRAZ, Sílvio. Música e Repetição: a diferença na composição contemporânea. São Paulo: Educ/FAPESP, 1998.

[10] XENAKIS, Iannis. Formalized Music. Revised Edition. Stuyvesant, NY: Pendragon Press, 1992.

[11] POUSSEUR, Henri. Apoteose de Rameau. Trad. Flo Menezes e Mauricio Oliveira Santos. Seleção de textos, prefácio e notas críticas de Flo Menezes. São Paulo: Ed. UNESP, 2009.

[12] TENNEY, J.; POLANSKY, L. Temporal Gestalt Perception in Music. Journal of Music Theory, vol. 24, $\mathrm{n}^{\circ}$ 2, pp. 205-241, 1980.

[13] Meta+Hodus. Ed. 2. Oakland: Frog Peak Music 1988.

[14] BOULEZ, Pierre. A música hoje. Ed. 3. São Paulo: Perspectiva, 2016. 